\title{
Combination of Bevacizumab and Acyclic Retinoid Inhibits the Growth of Hepatocellular Carcinoma Xenografts
}

\author{
Masaya Kubota, Masahito SHIMIzU*, Atsushi BABA, Tomohiko OHNO, \\ Takahiro KocHI, Yohei SHIRAKAMI and Hisataka MORIWAKI \\ Department of Gastroenterology/Internal Medicine, Gifu University Graduate School of Medicine, \\ Gifu 501-1194, Japan \\ (Received February 18, 2014)
}

\begin{abstract}
Summary The prognosis of patients with hepatocellular carcinoma (HCC) is poor and the development of effective treatments for this malignancy, including combination chemotherapy, is required. This study examined the possible combined inhibitory effects of bevacizumab, an anti-vascular endothelial growth factor monoclonal antibody, and acyclic retinoid (ACR), which can prevent the development of HCC, on the growth of Huh7 human HCC cells. Xenograft tumors were produced by subcutaneously injecting Huh7 cells into nude mice. Starting $1 \mathrm{wk}$ after the tumor cell injection, the mice were treated with bevacizumab alone $(5 \mathrm{mg} / \mathrm{kg}$ body weight, subcutaneous injection, twice a week), ACR alone (given in a diet containing $0.03 \%$ ), or their combination for $6 \mathrm{wk}$, and the effects of these regimens on xenograft growth were examined. Combined treatment with bevacizumab plus ACR significantly suppressed the growth of Huh7 xenografts. The combination of these agents significantly inhibited the phosphorylation of the Akt protein in tumor tissues. With combination therapy, the population of Ki-67-positive cells in xenografts decreased, while that of TUNEL-positive cells increased. The combination of bevacizumab and ACR exerts growth-suppressing effects on HCC cells by inhibiting cell proliferation and inducing apoptosis. This combination might be an effective regimen for the treatment of HCC.
\end{abstract}

Key Words bevacizumab, acyclic retinoid, hepatocellular carcinoma xenografts, cell proliferation, apoptosis

Hepatocellular carcinoma (HCC) is a major healthcare problem worldwide because of its high prevalence and mortality. Conventional chemotherapy for HCC has a limited curative effect and, therefore, there is an urgent need to develop more effective chemotherapeutic regimens for this malignancy (1). It is well known that HCC is a hypervascular tumor and that its growth depends on angiogenesis. For instance, the expression levels of vascular endothelial growth factor (VEGF), one of the most dominant proangiogenic growth factors in the tumor microenvironment (2), and its receptor (VEGFR) are significantly associated with angiogenic activity, tumor progression, and poor prognosis of HCC (3-6). Therefore, anti-angiogenic therapy using specific agents that can inhibit the activation of the VEGF-VEGFR axis may be beneficial in HCC (7). Recently, sorafenib, a multikinase inhibitor that blocks VEGF-VEGFR signaling, has been approved and widely used for the treatment of advanced HCC (8).

Bevacizumab is a recombinant, humanized monoclonal antibody that targets VEGF, and is used for the treatment of advanced-stage colorectal, lung, breast, and brain cancers (9). Phase II clinical trials have demonstrated the efficacy of bevacizumab when used as a

*To whom correspondence should be addressed.

E-mail: shimim-gif@umin.ac.jp single agent or in combination with cytotoxic or molecular-targeted agents for the treatment of advanced HCC $(10,11)$. The administration of bevacizumab significantly improved the survival of mice bearing human HCC tumors orthotopically transplanted in the liver (12). Moreover, several preclinical animal studies have indicated that the anti-tumor effect of bevacizumab on HCC is enhanced when it is combined with other chemotherapeutic agents (13-15). These findings suggest that the use of bevacizumab either as a single agent or in combination with other agents may be a promising strategy for the treatment of HCC.

Acyclic retinoid $(\mathrm{ACR})$ is a synthetic retinoid that can inhibit the growth of HCC-derived cells by inducing apoptosis (16). The administration of ACR reduced the incidence of post-therapeutic recurrence of HCC and improved the survival rate of patients with this malignancy $(17,18)$. Moreover, ACR acts synergistically with various anti-tumor and cancer preventive agents by suppressing growth and inducing apoptosis in human HCC cells (19-26). These findings suggest that combination therapy using ACR may be an effective approach to inhibit the growth of HCC cells $(27,28)$. In this study, we investigated the potential combined inhibitory effects of bevacizumab and ACR on the growth of Huh7 human HCC xenografts in BALB/c nude (nude) mice and the possible mechanisms involved. 
A

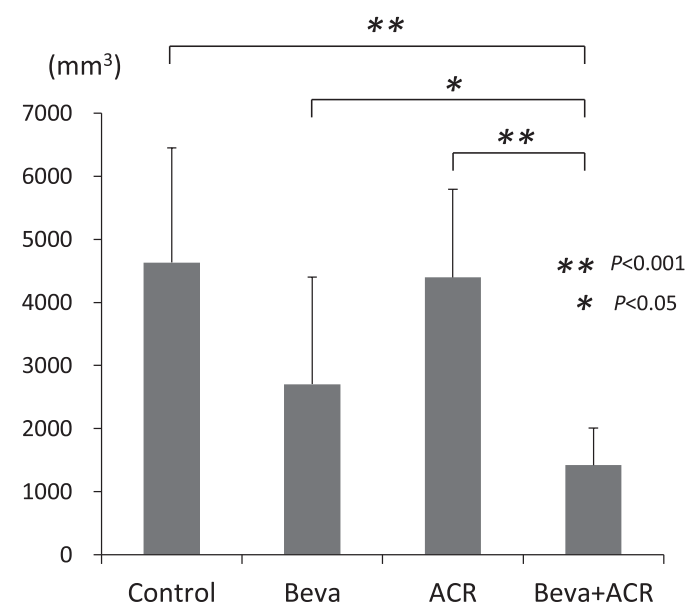

B

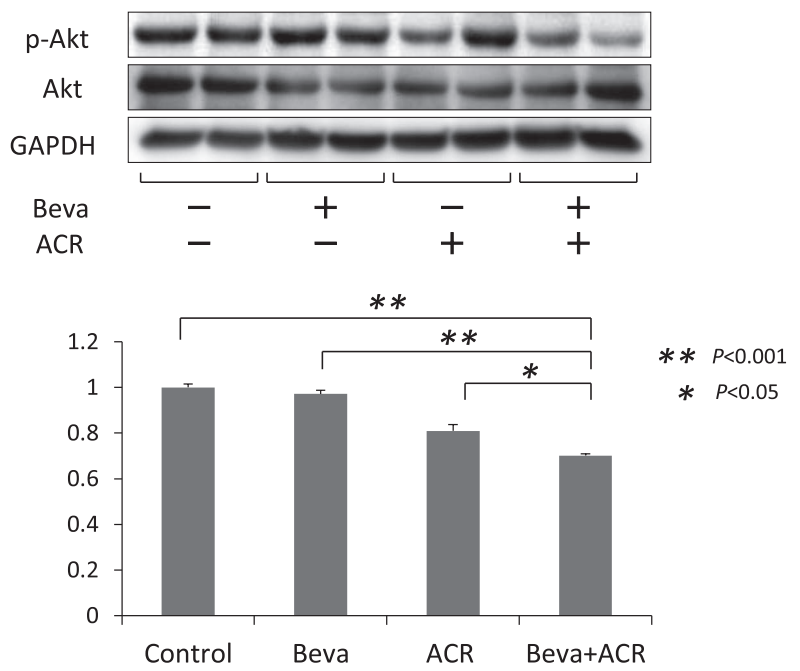

Fig. 1. Effects of the combination of bevacizumab plus ACR on the growth of Huh7 xenografts and on the levels of Akt phosphorylation in the xenografts. BALB/c nude mice were subcutaneously injected with $5 \times 10^{6}$ Huh7 cells. One week after the injection, the mice were divided into 4 groups and treated as follows for 6 wk: group 1, untreated control group; group 2, bevacizumab-treated group; group 3, ACR-treated group; and group 4, bevacizumab and ACR-treated group. (A) The volumes of xenografts, which were excised from each animal at the termination of the experiment, are represented. (B) Total proteins were extracted from xenografts, and equivalent amounts of proteins were examined by Western blot analysis. Protein samples from 2 different mice in each group are shown in the upper panel. The intensities of blots were quantitated by densitometry (lower panel). An antibody to GAPDH served as a loading control. Repeat Western blots gave similar results. Beva, bevacizumab. Bars, SD. * $p<0.05,{ }^{* *} p<0.001$.

\section{Materials and Methods}

Materials, animals, and cell line. Bevacizumab was purchased from Chugai Pharmaceutical Co. Ltd. (Tokyo, Japan). ACR (NIK-333) was supplied by Kowa Pharmaceutical Co. Ltd. (Tokyo, Japan). Nude mice $(n=48)$ were purchased from Charles River Japan (Tokyo, Japan). Huh7 cells were obtained from the Japanese Cancer Research Resources Bank (Tokyo, Japan).

Experimental procedure. At $6 \mathrm{wk}$ of age, xenograft tumors were produced by the subcutaneous injection of $5 \times 10^{6} \mathrm{Huh} 7$ cells in $200 \mu$ Linto the flanks of nude mice (29). One week after tumor cell injection, the mice were divided into the following 4 groups (12 mice per group): untreated control (group 1), bevacizumab alone (group 2), ACR alone (group 3), and bevacizumab plus ACR (group 4). Thereafter, mice were injected with bevacizumab ( $5 \mathrm{mg} / \mathrm{kg}$ body weight, groups 2 and 4 ) or saline (groups 1 and 3) subcutaneously twice a week for $6 \mathrm{wk}$. The dosage of bevacizumab was determined according to a previous study (14). Groups 1 and 2 were fed the basal diet CRF-1 (Oriental Yeast Co., Ltd., Tokyo, Japan) throughout the experiment. At $7 \mathrm{wk}$ of age, groups 3 and 4 were initiated on a basal diet containing $0.03 \%$ ACR for the next 6 wk. At 13 wk of age, xenografts were removed at sacrifice and the tumor volume was calculated using the formula: largest diameter $\times$ (smallest diameter $)^{2} \times 0.5$.

Immunohistochemical analysis of Ki-67. The excised xenografts were fixed in $10 \%$ buffered formaldehyde and embedded in paraffin. An immunohistochemical analysis of Ki-67 was performed using the sections cut from the tissue blocks and an anti-Ki-67 antibody
(DAKO, Glostrup, Denmark) (30). In the immunostained sections, Ki-67-positive nuclei in the hepatocytes were counted and expressed as a Ki-67-labeling index (\%) by counting at least 500 hepatocytes in each section (total of 3,000 hepatocytes per mouse) (31).

TUNEL assay. The sections prepared from the paraffin-embedded xenograft blocks were used for terminal deoxynucleotidyl transferase-mediated dUTP nick-end labeling (TUNEL) assay to evaluate the induction of apoptosis. After hydrophilization with the removal of wax on the tissue, the sections were incubated at $37^{\circ} \mathrm{C}$ for $30 \mathrm{~min}$ with proteinase $\mathrm{K}$, and were stained using both the 4',6-diamidino-2-phenylindole (DAPI) and TUNEL methods with the In Situ Cell Death Detection Kit, Fluorescein (Roche Diagnostics, Indianapolis, IN) (24).

Protein extraction and Western blot analysis. Total protein was extracted from the xenografts of Huh7 cells and equivalent amounts of protein were examined by a Western blot analysis using specific antibodies. The primary antibodies for Akt, phosphorylated-Akt, and glyceraldehyde-3-phosphate dehydrogenase (GAPDH) were purchased from Cell Signaling Technology (Beverly, MA) (25). The intensities of the blots were quantified with NIH Image software ver. 1.62. An antibody to GAPDH served as a loading control.

RNA extraction and quantitative real-time reverse transcription polymerase chain reaction analysis. Total RNA was isolated from the xenografts of Huh7 cells using the RNAqueous-4PCR kit (Ambion Applied Biosystems, Austin, TX), and cDNA was synthesized from $0.2 \mu \mathrm{g}$ of total RNA using the SuperScript III First-Strand Syn- 
A
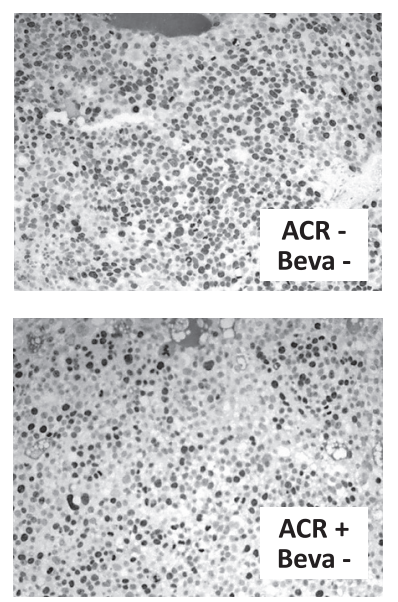

$\underline{\mathrm{Ki}-67 \mathrm{IHC}}$
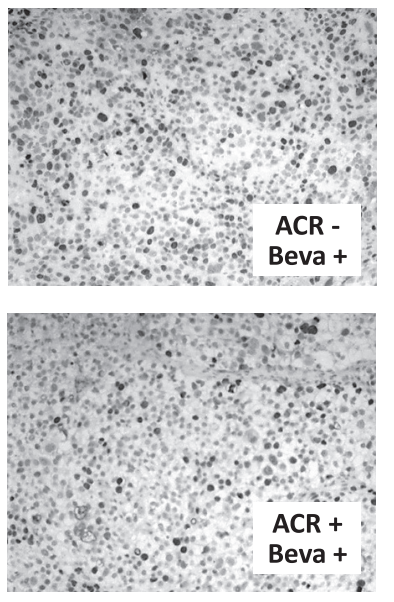

\section{B $\quad$ Ki-67-labeling index}

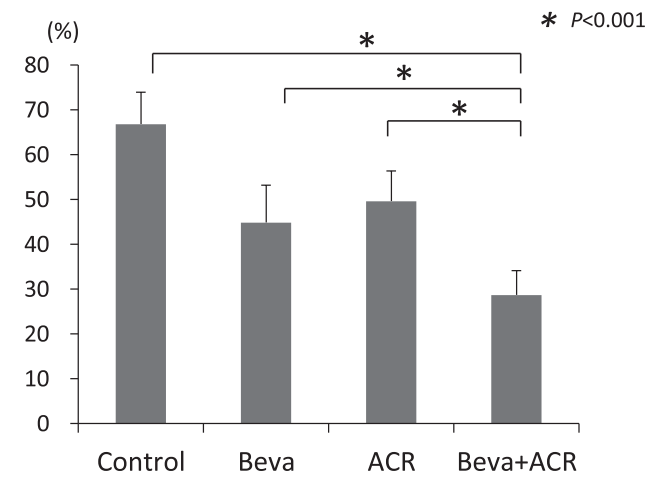

Fig. 2. Effects of the combination of bevacizumab plus ACR on cell proliferative activity of HCC xenografts. (A) Representative photographs of the Ki-67 immunohistochemical analysis of the HCC xenografts developed in the experimental mice.

(B) The Ki-67-labeling indices of the xenografts were determined by counting the Ki-67-positive nuclei in the xenografts. Bars, SD. ${ }^{*} p<0.001$.

A
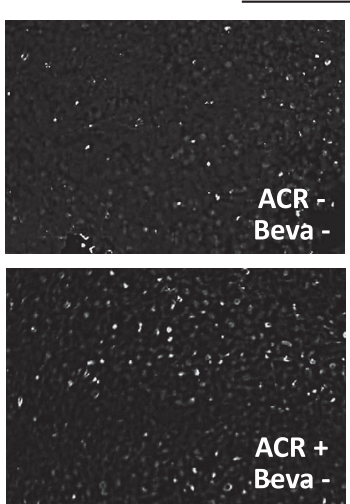

Beva -
TUNEL assay
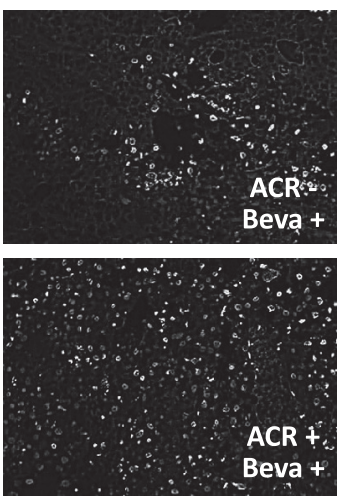

B Percentage of

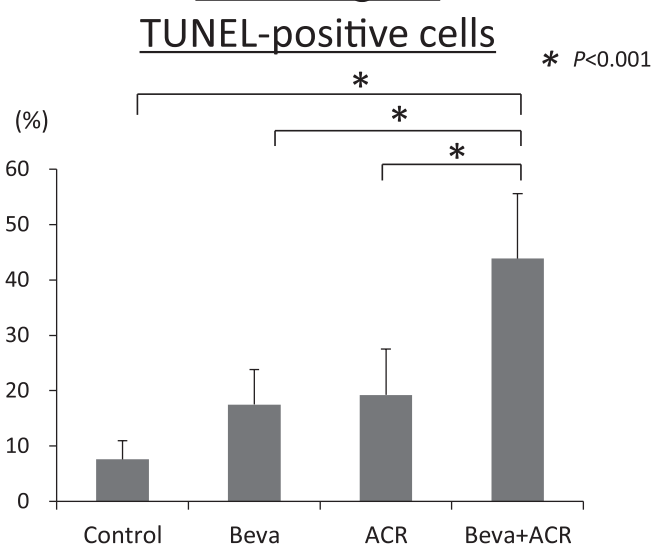

C $\underline{B A X / G A P D H}$

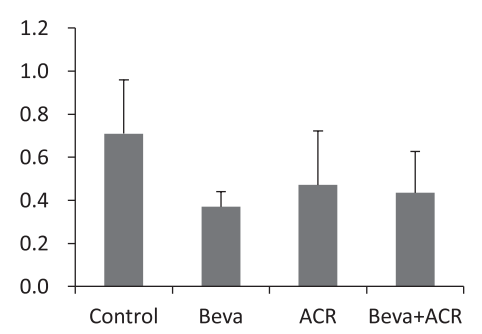

$\underline{\mathrm{BCl}-2 / \mathrm{GAPDH}}$

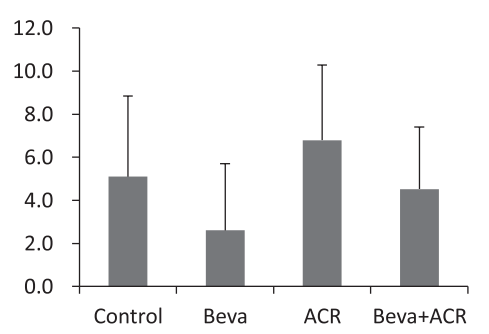

$\underline{\text { FOXO1/GAPDH }}$

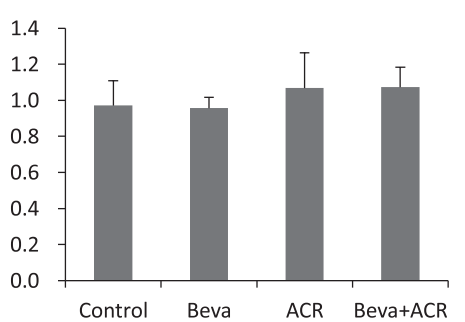

Fig. 3. Effects of the combination of bevacizumab plus ACR on the induction of apoptosis in HCC xenografts. (A) TUNEL assays were performed using sections prepared from the paraffin-embedded xenograft blocks. (B) TUNEL-positive cells were counted and expressed as a percentage of the DAPI-positive cell number (500 cells were counted in each section). (C) Tumor extracts were examined by quantitative RT-PCR analysis using specific primers for Bax, Bcl-2, and FOXO1. The expression levels of these genes were normalized to GAPDH expression. Bars, SD. * $p<0.001$.

thesis System (Invitrogen, Carlsbad, CA). Quantitative real-time reverse transcription-PCR (RT-PCR) analysis was performed using specific primers that amplified Bax, Bcl-2, Fork-head box O1 (FOXO1), and GAPDH genes, as described previously $(24,29,32,33)$.
Statistical analysis. The results are presented as the mean \pm SD. Statistical significance of the difference in mean values was assessed with one-way ANOVA, followed by Scheffe's $t$-test. 


\section{Results}

Combined treatment with bevacizumab plus ACR inhibits the growth of HCC xenografts and the phosphorylation of Akt in the xenografts

Figure 1A shows that neither bevacizumab alone ( $5 \mathrm{mg} / \mathrm{kg}$ body weight, subcutaneous injection) nor ACR alone (given in a diet containing $0.03 \%$ ) inhibited the growth of Huh7 xenografts, indicating that such doses of bevacizumab and ACR were insufficient to suppress HCC tumor growth in the present study. On the other hand, the volume of xenografts obtained from mice treated with bevacizumab plus ACR was significantly lower than that obtained from control untreated mice $(p<0.001)$, mice treated with bevacizumab alone $(p<0.05)$, or mice treated with ACR alone $(p<0.001)$. The expression levels of phosphorylated-Akt protein in HCC xenografts were significantly reduced in the bevacizumab plus ACR group compared to those in the control group $(p<0.001)$, the group treated with bevacizumab alone $(p<0.001)$, and the group treated with ACR alone $(p<0.05)$ (Fig. 1B).

Combined treatment with bevacizumab plus ACR inhibits the cell proliferative activity of HCC xenografts

An immunohistochemical analysis of Ki-67 was carried out to evaluate the inhibitory effects of bevacizumab and ACR on the cell proliferative activity of HCC xenografts. The Ki-67-labeling index of the xenografts was determined based on findings from the Ki-67-immunostained sections (Fig. 2A). The mean Ki-67-labeling index in the mice treated with the combination of bevacizumab plus ACR was significantly lower than that of the control untreated mice, mice treated with bevacizumab alone, or mice treated with ACR alone (Fig. 2B; $p<0.001$ for each comparison), indicating that combination treatment with these agents significantly inhibited the cell proliferative activity of xenografts.

Combined treatment with bevacizumab plus ACR induces apoptosis in HCC xenografts

The induction of apoptosis in HCC xenografts by bevacizumab and ACR was estimated using the TUNEL assays (Fig. 3A). Neither treatment with bevacizumab alone nor ACR alone increased the number of TUNELpositive cells in HCC xenografts. However, the number of these cells significantly increased when mice were treated with the combination of bevacizumab plus ACR (Fig. 3B; $p<0.001$ for each comparison), suggesting that the induction of apoptosis was markedly enhanced by the combination of these agents. Treatment with bevacizumab alone, ACR alone, or the combination of these agents did not affect the expression levels of Bax or Bcl-2 mRNA, both of which regulate apoptosis by controlling mitochondrial permeability (34). The expression level of FOXO1 mRNA, which is negatively regulated by Akt phosphorylation and is thus associated with the promotion of apoptosis $(35,36)$, was not altered by treatment with these agents when used either alone or simultaneously (Fig. 3C).

\section{Discussion}

Several clinical trials have suggested that bevaci- zumab may be a promising agent for the treatment of advanced HCC $(10,11)$. However, data supporting the ability of this antibody to improve the prognosis of HCC patients are insufficient at present. In addition, bevacizumab is known to have a number of severe adverse effects such as bleeding and thrombosis (37). Therefore, in considering the clinical application of bevacizumab for HCC treatment, the need to improve the efficacy and safety of this agent demands a prompt solution. We considered that combination therapy using bevacizumab and other agents may present such a solution by providing the potential for synergistic effects on the inhibition of tumor growth and permitting the use of lower drug dosages, consequently decreasing toxicity. Indeed, the results from Phase II trials targeting HCC patients suggest that bevacizumab may be more effective when combined with other agents $(10,11)$.

The present study clearly indicated that the combination of bevacizumab plus ACR causes potent inhibition of growth in human HCC xenografts through the inhibition of cell proliferation and induction of apoptosis in HCC cells. We consider the suppressive effect of this combination therapy on the tumor growth to be possibly associated with the inhibition of Akt phosphorylation because the activation of phosphatidylinositol 3-kinase (PI3K)/Akt signaling plays a critical role in the survival and proliferation of HCC cells and, therefore, targeting this signaling might be an effective strategy for the treatment of $\operatorname{HCC}(38,39)$. A recent experimental study showed that bevacizumab enhances the chemosensitivity of HCC by inhibiting the VEGF-PI3K/Akt signaling pathway (14). Phosphorylation of Akt is also one of the targets by which ACR suppresses the growth of HCC cells (26). In addition, ACR exerts synergistic growth inhibitive effects in HCC cells when it is simultaneously used with specific agents that inhibit the phosphorylation of Akt $(19,23,25,26)$.

In the present study, the combination of bevacizumab plus ACR significantly increased the number of TUNELpositive cells in HCC xenografts, whereas the expression levels of Bax, Bcl-2, and FOXO1 mRNA were not altered by this combination. These findings suggest that controlling mitochondrial permeability, which is regulated by the Akt/FOXO1 signaling pathway (40), is not the main mechanism of this combination, suggesting that other existing mechanisms induce apoptosis in HCC xenografts. For instance, the combination of bevacizumab plus ACR might induce apoptosis in HCC xenografts by directly inhibiting caspase- 9 activity being that this protease is a critical target of activated Akt for the resistance of apoptosis induction (41). Additional experiments are required to prove this speculation.

The results from previous studies $(19,24-26)$ show that combination of ACR plus specific agents inhibits the growth of HCC cells by regulating the expression levels of p $21^{\mathrm{CIP} 1}$ and cyclin D1, both of which control cell cycle progression (42). Therefore, we examined whether the combination of bevacizumab plus ACR affected the levels of p21 ${ }^{\mathrm{CIP} 1}$ and cyclin D1 in HCC xenografts, but the levels of these molecules were not altered by this 
combination (data not shown). In addition, treatment with this combination did not affect the expression level of FOXO1, which also controls the cell cycle by up-regulating the expression of $\mathrm{p} 21^{\mathrm{CIP} 1}$ (36). These findings suggest that controlling the cell cycle progression might not be a critical mechanism of this combination for growth suppression of HCC cells.

In addition to the inhibition of angiogenesis, bevacizumab is thought to exert its anti-tumor effects by normalizing tumor vasculature, which decreases the elevated interstitial pressure in the tumor and thus leads to improved drug delivery (43). Therefore, in the present study, bevacizumab may have enhanced the distribution of ACR across the capillaries to the tumor cells by normalizing tumor vasculature. Moreover, ACR is reported to exert anti-angiogenic effects by inhibiting the activation of VEGFR2 (44). A gene expression analysis using clinical samples also showed that ACR administration prevents the recurrence of HCC, at least in part by suppressing the expression of angiogenesis genes (45), suggesting that the VEGF-VEGFR axis might be a direct target of ACR. Therefore, future studies are required to determine whether the vessel phenotype is normalized and the activation of the VEGF-VEGFR axis is inhibited after combination treatment with bevacizumab plus ACR.

In conclusion, this study, together with previously reported results (13-15), indicates that combination therapy using bevacizumab as a key drug might be a promising regimen for the treatment of HCC. In a bevacizumab-based combination therapy regimen, ACR, which successfully improves HCC patient survival by preventing recurrence $(17,18)$, is a preferred partner for bevacizumab because the combination of these agents significantly suppresses the growth of HCC xenografts by inhibiting cell proliferation and inducing apoptosis in HCC cells.

\section{REFERENCES}

1) Thomas MB, Zhu AX. 2005. Hepatocellular carcinoma: the need for progress. J Clin Oncol 23: 2892-2899.

2) Ferrara N. 2002. VEGF and the quest for tumour angiogenesis factors. Nat Rev Cancer 2: 795-803.

3) Mise M, Arii S, Higashituji H, Furutani M, Niwano M, Harada T, Ishigami S, Toda Y, Nakayama H, Fukumoto M, Fujita J, Imamura M. 1996. Clinical significance of vascular endothelial growth factor and basic fibroblast growth factor gene expression in liver tumor. Hepatology 23: $455-464$.

4) Torimura T, Sata M, Ueno T, Kin M, Tsuji R, Suzaku K, Hashimoto O, Sugawara H, Tanikawa K. 1998. Increased expression of vascular endothelial growth factor is associated with tumor progression in hepatocellular carcinoma. Hum Pathol 29: 986-991.

5) Ng IO, Poon RT, Lee JM, Fan ST, Ng M, Tso WK. 2001. Microvessel density, vascular endothelial growth factor and its receptors Flt-1 and Flk-1/KDR in hepatocellular carcinoma. Am J Clin Pathol 116: 838-845.

6) Dhar DK, Naora H, Yamanoi A, Ono T, Kohno H, Otani H, Nagasue N. 2002. Requisite role of VEGF receptors in angiogenesis of hepatocellular carcinoma: a comparison with angiopoietin/Tie pathway. Anticancer Res 22:
379-386.

7) Zhu AX, Duda DG, Sahani DV, Jain RK. 2011. HCC and angiogenesis: possible targets and future directions. Nat Rev Clin Oncol 8: 292-301.

8) Forner A, Llovet JM, Bruix J. 2012. Hepatocellular carcinoma. Lancet 379: 1245-1255.

9) Presta LG, Chen H, O'Connor SJ, Chisholm V, Meng YG, Krummen L, Winkler M, Ferrara N. 1997. Humanization of an anti-vascular endothelial growth factor monoclonal antibody for the therapy of solid tumors and other disorders. Cancer Res 57: 4593-4599.

10) Fang P, Hu JH, Cheng ZG, Liu ZF, Wang JL, Jiao SC. 2012. Efficacy and safety of bevacizumab for the treatment of advanced hepatocellular carcinoma: a systematic review of phase II trials. PLoS One 7: e49717.

11) Wenger JB, Santos N, Liu Y, Dallas J, Subbiah S, Hochwald S, Huang EH, Dang DT, Allegra CJ, Luesch H, Dang LH. 2011. Can we develop effective combination antiangiogenic therapy for patients with hepatocellular carcinoma? Oncol Rev 5: 177-184.

12) Finn RS, Bentley G, Britten CD, Amado R, Busuttil RW. 2009. Targeting vascular endothelial growth factor with the monoclonal antibody bevacizumab inhibits human hepatocellular carcinoma cells growing in an orthotopic mouse model. Liver Int 29: 284-290.

13) Huynh H, Chow PK, Palanisamy N, Salto-Tellez M, Goh BC, Lee CK, Somani A, Lee HS, Kalpana R, Yu K, Tan PH, Wu J, Soong R, Lee MH, Hor H, Soo KC, Toh HC, Tan P. 2008. Bevacizumab and rapamycin induce growth suppression in mouse models of hepatocellular carcinoma. J Hepatol 49: 52-60.

14) Xiong YQ, Sun HC, Zhu XD, Zhang W, Zhuang PY, Zhang JB, Xu HX, Kong LQ, Wu WZ, Qin LX, Tang ZY. 2011. Bevacizumab enhances chemosensitivity of hepatocellular carcinoma to adriamycin related to inhibition of survivin expression. J Cancer Res Clin Oncol 137: 505-512.

15) Zhou F, Hu J, Shao JH, Zou SB, Shen SL, Luo ZQ. 2012. Metronomic chemotherapy in combination with antiangiogenic treatment induces mosaic vascular reduction and tumor growth inhibition in hepatocellular carcinoma xenografts. J Cancer Res Clin Oncol 138: 1879-1890.

16) Suzui M, Masuda M, Lim JT, Albanese C, Pestell RG, Weinstein IB. 2002. Growth inhibition of human hepatoma cells by acyclic retinoid is associated with induction of p21(CIP1) and inhibition of expression of cyclin D1. Cancer Res 62: 3997-4006.

17) Muto Y, Moriwaki H, Ninomiya M, Adachi S, Saito A, Takasaki KT, Tanaka T, Tsurumi K, Okuno M, Tomita E, Nakamura T, Kojima T. 1996. Prevention of second primary tumors by an acyclic retinoid, polyprenoic acid, in patients with hepatocellular carcinoma. Hepatoma Prevention Study Group. N Engl J Med 334: 1561-1567.

18) Muto Y, Moriwaki H, Saito A. 1999. Prevention of second primary tumors by an acyclic retinoid in patients with hepatocellular carcinoma. $N$ Engl J Med 340: 1046-1047.

19) Tatebe H, Shimizu M, Shirakami Y, Sakai H, Yasuda Y, Tsurumi H, Moriwaki H. 2009. Acyclic retinoid synergises with valproic acid to inhibit growth in human hepatocellular carcinoma cells. Cancer Lett 285: 210-217.

20) Obora A, Shiratori Y, Okuno M, Adachi S, Takano Y, Matsushima-Nishiwaki R, Yasuda I, Yamada Y, Akita 
K, Sano T, Shimada J, Kojima S, Okano Y, Friedman SL, Moriwaki H. 2002. Synergistic induction of apoptosis by acyclic retinoid and interferon-beta in human hepatocellular carcinoma cells. Hepatology 36: 1115-1124.

21) Shimizu M, Suzui M, Deguchi A, Lim JT, Xiao D, Hayes JH, Papadopoulos KP, Weinstein IB. 2004. Synergistic effects of acyclic retinoid and OSI-461 on growth inhibition and gene expression in human hepatoma cells. Clin Cancer Res 10: 6710-6721.

22) Kanamori T, Shimizu M, Okuno M, Matsushima-Nishiwaki R, Tsurumi H, Kojima S, Moriwaki H. 2007. Synergistic growth inhibition by acyclic retinoid and vitamin K2 in human hepatocellular carcinoma cells. Cancer Sci 98: $431-437$.

23) Tatebe H, Shimizu M, Shirakami Y, Tsurumi H, Moriwaki H. 2008. Synergistic growth inhibition by 9-cis-retinoic acid plus trastuzumab in human hepatocellular carcinoma cells. Clin Cancer Res 14: 2806-2812.

24) Ohno T, Shirakami Y, Shimizu M, Kubota M, Sakai H, Yasuda Y, Kochi T, Tsurumi H, Moriwaki H. 2012. Synergistic growth inhibition of human hepatocellular carcinoma cells by acyclic retinoid and GW4064, a farnesoid X receptor ligand. Cancer Lett 323: 215-222.

25) Shimizu M, Shirakami Y, Sakai H, Iwasa J, Shiraki M, Takai K, Naiki T, Moriwaki H. 2012. Combination of acyclic retinoid with branched-chain amino acids inhibits xenograft growth of human hepatoma cells in nude mice. Hepatol Res 42: 1241-1247.

26) Baba A, Shimizu M, Ohno T, Shirakami Y, Kubota M, Kochi T, Terakura D, Tsurumi H, Moriwaki H. 2013. Synergistic growth inhibition by acyclic retinoid and phosphatidylinositol 3-kinase inhibitor in human hepatoma cells. BMC Cancer 13: 465.

27) Shimizu M, Imai K, Takai K, Moriwaki H. 2012. Role of acyclic retinoid in the chemoprevention of hepatocellular carcinoma: basic aspects, clinical applications, and future prospects. Curr Cancer Drug Targets 12: 1119-1128.

28) Shimizu M, Shirakami Y, Imai K, Takai K, Moriwaki H. 2012. Acyclic retinoid in chemoprevention of hepatocellular carcinoma: Targeting phosphorylated retinoid $\mathrm{X}$ receptor-alpha for prevention of liver carcinogenesis. J Carcinog 11: 11.

29) Shirakami Y, Shimizu M, Adachi S, Sakai H, Nakagawa T, Yasuda Y, Tsurumi H, Hara Y, Moriwaki H. 2009. (-)-Epigallocatechin gallate suppresses the growth of human hepatocellular carcinoma cells by inhibiting activation of the vascular endothelial growth factorvascular endothelial growth factor receptor axis. Cancer Sci 100: 1957-1962.

30) Quaglia A, McStay M, Stoeber K, Loddo M, Caplin M, Fanshawe T, Williams G, Dhillon A. 2006. Novel markers of cell kinetics to evaluate progression from cirrhosis to hepatocellular carcinoma. Liver Int 26: 424-432.
31) Iwasa J, Shimizu M, Shiraki M, Shirakami Y, Sakai H, Terakura Y, Takai K, Tsurumi H, Tanaka T, Moriwaki H. 2010. Dietary supplementation with branched-chain amino acids suppresses diethylnitrosamine-induced liver tumorigenesis in obese and diabetic C57BL/KsJ-db/db mice. Cancer Sci 101: 460-467.

32) Monga J, Pandit S, Chauhan RS, Chauhan CS, Chauhan SS, Sharma M. 2013. Growth inhibition and apoptosis induction by (+)-Cyanidan-3-ol in hepatocellular carcinoma. PLoS One 8: e68710.

33) Miao H, Zhang Y, Lu Z, Liu Q, Gan L. 2012. FOXO1 involvement in insulin resistance-related pro-inflammatory cytokine production in hepatocytes. Inflamm Res 61: 349-358.

34) Brenner D, Mak TW. 2009. Mitochondrial cell death effectors. Curr Opin Cell Biol 21: 871-877.

35) Essaghir A, Dif N, Marbehant CY, Coffer PJ, Demoulin JB. 2009. The transcription of FOXO genes is stimulated by FOXO3 and repressed by growth factors. J Biol Chem 284: 10334-10342.

36) Greer EL, Brunet A. 2005. FOXO transcription factors at the interface between longevity and tumor suppression. Oncogene 24: 7410-7425.

37) Di Fiore F, Van Cutsem E. 2009. Acute and long-term gastrointestinal consequences of chemotherapy. Best Pract Res Clin Gastroenterol 23: 113-124.

38) Zhou Q, Lui VW, Yeo W. 2011. Targeting the PI3K/Akt/ mTOR pathway in hepatocellular carcinoma. Future Oncol 7: 1149-1167.

39) Llovet JM, Bruix J. 2008. Molecular targeted therapies in hepatocellular carcinoma. Hepatology 48: 1312-1327.

40) Amin AR, Paul RK, Thakur VS, Agarwal ML. 2007. A novel role for $\mathrm{p} 73$ in the regulation of Akt-Foxola-Bim signaling and apoptosis induced by the plant lectin, Concanavalin A. Cancer Res 67: 5617-5621.

41) Cardone MH, Roy N, Stennicke HR, Salvesen GS, Franke TF, Stanbridge E, Frisch S, Reed JC. 1998. Regulation of cell death protease caspase-9 by phosphorylation. Science 282: 1318-1321.

42) Liang J, Slingerland JM. 2003. Multiple roles of the PI3K/PKB (Akt) pathway in cell cycle progression. Cell Cycle 2: 339-345.

43) Jain RK. 2005. Normalization of tumor vasculature: an emerging concept in antiangiogenic therapy. Science 307: $58-62$.

44) Komi Y, Sogabe Y, Ishibashi N, Sato Y, Moriwaki H, Shimokado K, Kojima S. 2010. Acyclic retinoid inhibits angiogenesis by suppressing the MAPK pathway. Lab Invest 90: 52-60.

45) Honda M, Yamashita T, Arai K, Sakai Y, Sakai A, Nakamura M, Mizukoshi E, Kaneko S. 2013. Peretinoin, an acyclic retinoid, improves the hepatic gene signature of chronic hepatitis $\mathrm{C}$ following curative therapy of hepatocellular carcinoma. BMC Cancer 13: 191. 